\title{
Gastroesophageal reflux disease, irritable bowel syndrome and functional dyspepsia as overlapping conditions: focus on effect of trimebutine
}

\section{Jannis Kountouras ${ }^{a}$, Michael Doulberis ${ }^{\mathrm{a}, \mathrm{b}}$, Apostolis Papaefthimiou ${ }^{a, c}$, Stergios A. Polyzos ${ }^{a, d}$}

Aristotle University of Thessaloniki, Ippokration Hospital, Thessaloniki, Macedonia, Greece; University Hospital Inselspital, Bern, Switzerland; 401 General Military Hospital of Athens, Greece

In their comprehensive review, de Bortoli et al [1] aimed to discuss the coexistence of gastroesophageal reflux disease (GERD) with irritable bowel syndrome (IBS) and functional dyspepsia (FD) in the same patients and to evaluate the impact of diverse GERD treatments on the health-related quality of life (HR-QOL) of these patients; they mentioned 2 trials that reported resolution of the IBS symptoms in $20-40 \%$ of patients after proton pump inhibitor (PPI) therapy [1]. In one of these trials, we mainly observed [2] that trimebutine maleate is effective in the aforementioned overlapping disorders.

Relative data indicate that the prevalence of overlaps of FD and/or IBS in GERD, GERD and/or IBS in FD, and GERD and/or FD in IBS is $46.9 \%, 47.6 \%$ and $34.4 \%$, respectively, with worsened HR-QOL [3]. Moreover, Helicobacter pylori (H. pylori) infection could also be involved in GERD pathophysiology, at least in some national studies, as well as in FD and IBS patients $[4,5]$.

In this regard, our own preliminary data [2], also mentioned by the authors [1], showed that $H$. pylori is common in patients with GERD-IBS-FD and/or erosive esophagitis, while H. pylori eradication plus PPI and/or trimebutine regimens offer improvement in HR-QOL, mainly in patients who receive trimebutine. In a subsequent study, we confirmed our preliminary data [6], signifying the effectiveness of trimebutine in these overlapping populations.

In overlapping disorders trimebutine could act: as a modulator of gastrointestinal tract motility being a promising candidate for treatment of hypermotility and hypomotility disorders [7]; by hastening gastric emptying, shortening the lag period, causing a premature phase III of the migrating motor complexes in the gut and controlling colonic contractile action $[2,8]$; by inducing release of gastrointestinal agents such as motilin; by modulating visceral sensitivity; by ameliorating symptoms such as diarrhea and abdominal pain $[2,8]$; and as a possible antimicrobial agent against bacteria that could trigger post-infectious functional gastrointestinal disorders $[9,10]$, thereby requiring additional relative investigation.

\section{References}

1. de Bortoli N, Tolone S, Frazzoni M, et al. Gastroesophageal reflux disease, functionaldyspepsia andirritablebowelsyndrome:common overlapping gastrointestinal disorders. Ann Gastroenterol 2018; 31:639-648.

2. Kountouras J, Chatzopoulos D, Zavos C, Boura P, Venizelos J, Kalis A. Efficacy of trimebutine therapy in patients with gastroesophageal reflux disease and irritable bowel syndrome. Hepatogastroenterology 2002;49:193-197.

3. Kaji M, Fujiwara Y, Shiba M, et al. Prevalence of overlaps between GERD, FD and IBS and impact on health-related quality of life. J Gastroenterol Hepatol 2010;25:1151-1156.

4. Kountouras J, Zavos C, Polyzos SA, Katsinelos P. Helicobacter pylori infection and gastroesophageal reflux disease - Barrett's esophagus sequence "dilemma". Ann Gastroenterol 2015;28:153.

5. Su YC, Wang WM, Wang SY, et al. The association between Helicobacter pylori infection and functional dyspepsia in patients with irritable bowel syndrome. Am J Gastroenterol 2000; 95:1900-1905.

6. Kountouras J, Zavos C, Chatzopoulos D. H pylori infection and reflux oesophagitis. Gut 2004;53:912.

7. Lee HT, Kim BJ. Trimebutine as a modulator of gastrointestinal motility. Arch Pharm Res 2011;34:861-864.

8. Hussain Z, Jung DH, Lee YJ, Park H. The effect of trimebutine on the overlap syndrome model of Guinea pigs. J Neurogastroenterol Motil 2018;24:669-675.

9. Kruszewska H, Zareba T, Tyski S. Examination of antimicrobial activity of selected non-antibiotic drugs. Acta Pol Pharm 2004;61(Suppl):18-21.

10. Kountouras J, Sofianou D, Gavalas E, et al. Trimebutine as a potential antimicrobial agent: a preliminary in vitro approach. Hippokratia 2012;16:347-349.

aDepartment of Medicine, Second Medical Clinic, Aristotle University of Thessaloniki, Ippokration Hospital, Thessaloniki, Macedonia, Greece (Jannis Kountouras, Michael Doulberis, Apostolis Papaefthimiou,

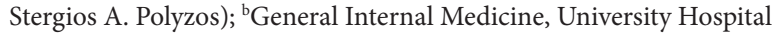
Inselspital, Bern, Switzerland (Michael Doulberis); 'Gastroenterology, 401 General Military Hospital of Athens, Greece; (Apostolis Papaefthimiou); ${ }^{\mathrm{d}}$ First Department of Pharmacology, Department of Medicine, Aristotle University of Thessaloniki, Ippokration Hospital, Thessaloniki, Macedonia, Greece (Stergios A. Polyzos)

Conflict of Interest: None

Correspondence to: Jannis Kountouras, MD, PhD, Professor of Medicine, Gastroenterologist, 8 Fanariou St, Byzantio, 551 33, Thessaloniki, Macedonia, Greece,

e-mail: jannis@auth.gr, ancoratus2010@gmail.com

Received 17 January 2019; accepted 30 January 2019; published online 18 February 2019

DOI: https://doi.org/10.20524/aog.2019.0366 